\title{
Vinte Anos de Estudo sobre o Reconhecimento de Palavras em Crianças Falantes do Português: Uma Revisão de Literatura
}

\author{
Twenty Years of Studies on Word Recognition with Portuguese \\ Speaking Children: A Literature Review
}

\author{
Patrícia Silva Lúcio* \& Ângela Maria Vieira Pinheiro \\ Universidade Federal de Minas Gerais, Belo Horizonte, Brasil
}

\begin{abstract}
Resumo
A presente revisão de literatura busca apresentar as principais conclusões dos estudos conduzidos na área de reconhecimento de palavras com crianças falantes do português e levantar os pontos teóricos e metodológicos que ainda merecem atenção dos pesquisadores. A ênfase do trabalho recai sobre a tarefa de leitura em voz alta de palavras isoladas feita por crianças em fase de aquisição da leitura. Três grandes conclusões foram tiradas: (a) os diversos estudos em língua portuguesa confirmam, de maneira geral, os postulados do modelo de dupla-rota da leitura; (b) as crianças falantes do português parecem utilizar preferencialmente a estratégia fonológica no início da aprendizagem da leitura, a qual vai sendo gradualmente substituída pelo uso prioritário da estratégia lexical; e (c) questões metodológicas têm interferido nos resultados encontrados pelos diferentes pesquisadores, as quais se relacionam à forma de aplicação da tarefa de leitura, ao tipo de análise estatística utilizada e à direção (do ponto de vista da leitura ou da escrita) e ao número de categorias consideradas na classificação de regularidade das palavras.

Palavras-chave: Revisão de Literatura; Reconhecimento de palavras; Efeito de regularidade; Frequência.

Abstract

The present work attempts to review the main conclusions of the studies carried out in the area of word recognition with Portuguese speaking children and to raise theoretical and methodological issues that still require the attention of researchers. The emphasis of this work is centered on the word reading aloud task performed by children in the process of reading acquisition. Three major conclusions were derived from the review: (a) generally, the collection of studies in Portuguese language confirm the assumptions of the dual-route model of word recognition; (b) Portuguese speaking children seem to prefer to use the phonological strategy in the beginning of the reading process, which is then gradually substituted by the preferential use of the lexical strategy; and (c) methodological issues have interfered in the results found by different researchers, and these are related to the manner of application of the reading task, the type of statistical analysis carried out and to the direction (from the reading or spelling standpoint) and number of categories considered in the classification of regularity of the words.

Keywords: Literature review; Word recognition; Regularity effect; Frequency.
\end{abstract}

Já se passaram 20 anos desde que foi conduzido o primeiro estudo (Pinheiro, 1989 - tese de doutorado publicada em 1995, 2008) que buscou investigar a aplicabilidade do modelo de dupla-rota na leitura em voz alta de palavras isoladas em crianças falantes do português brasileiro. Tal empreitada foi conduzida a partir da investigação dos efeitos de frequência, regularidade, ex-

\footnotetext{
* Endereço para correspondência: Rua Costa Rica, n 455, Bairro Nações Unidas, Sabará, MG, Brasil, CEP: 34590-380. Tels.: (31) 3499-6268 (Universidade Federal de Minas Gerais); (31) 3671-9452 (residencial), (31) 9637-8085 (celular). E-mail: pslucio@gmail.com Esta pesquisa é parte da dissertação de mestrado da primeira autora, orientada pela segunda autora. Agradecemos ao apoio financeiro da Fundação de Amparo à Pesquisa do Estado de Minas Gerais (FAPEMIG) e do Conselho Nacional de Desenvolvimento Científico e Tecnológico $(\mathrm{CNPq})$
}

tensão e de lexicalidade no tempo de processamento e nos erros cometidos na leitura e por uma detalhada análise dos tipos de erros ${ }^{1}$. Após este estudo pioneiro, vários pesquisadores se dedicaram à investigação desses efeitos tanto no português brasileiro (p. ex., A. G. S. Capovilla, Capovilla, \& Macedo 1998; Godoy, 2005; C. Justi \& Justi, 2009; Lúcio, 2008 - dissertação de mestrado publicada em Lúcio, Pinheiro, \& Nascimento, 2010; Pinheiro, Lúcio, \& Silva, 2008; Salles, 2001 - dissertação de mestrado publicada em Salles \& Parente, 2002; Salles, 2005 - tese

\footnotetext{
${ }^{1}$ Não trataremos aqui sobre a análise dos tipos de erros que, como os efeitos de leitura, podem não só sinalizar o tipo de processo utilizado pelo leitor, como também indicar a mudança de processos ao longo do desenvolvimento. Para uma revisão dessa parte do estudo de reconhecimento de palavras, ver Pinheiro, Lúcio e $\mathrm{Cu}$ nha (2008).
} 
de doutorado publicada em Salles \& Parente, 2007) quanto no de Portugal (p. ex., Sucena \& Castro, 2005; Viana, Pereira, \& Teixeira, 2003). Nesse sentido, torna-se evidente a existência de um grande volume de dados desde a publicação daquele primeiro trabalho, tendo, portanto, esta revisão o objetivo de sintetizar os achados produzidos até o presente momento pelas pesquisas que buscaram avaliar os referidos efeitos na leitura em voz alta de palavras de crianças falantes do português. Pretende-se, desta forma, apresentar as principais conclusões dos estudos e levantar os pontos teóricos e metodológicos que ainda merecem atenção dos pesquisadores estudiosos na área de reconhecimento de palavras, destacando-se aqueles relacionados à emergência do efeito de regularidade.

\section{Uma Breve Descrição sobre os Efeitos na Leitura}

Os efeitos na leitura podem ser definidos como a vantagem que as características dos estímulos ou dos participantes produzem na precisão e/ou no tempo de processamento da leitura. No primeiro caso, o efeito se deve a determinadas características dos itens, enquanto que, no segundo, o efeito se deve a diferenças inter-individuais (como a idade ou o sexo). Em relação à tarefa de leitura de voz alta de palavras isoladas, a manipulação das características das palavras produz efeitos que servem para avaliar quais os componentes do modelo de dupla-rota que estão sendo utilizados pelo leitor (Coltheart, Rastle, Perry, Langdon, \& Ziegler, 2001). Tal manipulação afeta a leitura realizada tanto por crianças em fase de aquisição da leitura, quanto por aqueles leitores que apresentam dislexia (seja ela do desenvolvimento ou adquirida). As propriedades das palavras mais comumente manejadas para este fim são a frequência (efeito de frequência), a regularidade (efeito de regularidade), o comprimento (que produz o efeito de extensão) e a lexicalidade (ou seja, se a palavra existe na língua ou não, o que produz o efeito lexical). Apesar de a presença destes efeitos na leitura estar intimamente ligada ao nível de proficiência do leitor (pois, em geral, aparecem apenas na leitura de iniciantes), é possível afirmar que o esperado é que ocorram vantagens (maior correção/rapidez) na leitura das palavras familiares em relação às não-familiares, das palavras regulares em relação às irregulares, das palavras menores em relação às de maior comprimento e das palavras existentes na língua em relação às palavras sem significado. Os efeitos de regularidade e de extensão são indicadores do uso de processo fonológico na leitura, enquanto que os efeitos de frequência (este dentro do limite esperado) e lexical são indicadores do uso do processo lexical. Uma descrição mais detalhada dos efeitos na leitura em voz alta de palavras, assim como do modelo de dupla-rota, pode ser encontrada em Coltheart et al. (2001), Godoy (2005), Pinheiro (2008) e Pinheiro e Rothe-Neves (2001).

\section{Método}

A presente revisão se concentra em estudos empíricos que relataram os efeitos de frequência, de regularidade, de extensão e/ou de lexicalidade na leitura em voz alta de palavras isoladas feitas por crianças em fase de aquisição da leitura falantes do português ${ }^{2}$. O levantamento considerou as bases de dados Google Acadêmico, Scielo, Lilacs, PEPsic e banco de teses e dissertações da Coordenação de Aperfeiçoamento de Pessoal de Nível Superior (CAPES). Foram utilizadas as palavras-chaves: "leitura", "frequência", "regularidade", "extensão", "lexical", "criança" e "Portugal". Ao todo, foram encontrados 46 trabalhos, dos quais foram excluídas as revisões de literatura, os que não tratavam da tarefa de leitura em voz alta, os que não tratavam dos efeitos na leitura, os que apresentavam adolescentes ou adultos na amostra e os trabalhos que reportaram dados apenas de crianças disléxicas ou com dificuldades de aprendizagem. A presente revisão sintetiza os achados de 18 trabalhos de pesquisadores brasileiros e quatro de pesquisadores portugueses.

\section{Os Principais Resultados de Estudos em Língua Portuguesa}

No primeiro estudo brasileiro sobre reconhecimento de palavras, Pinheiro $(1995,2008)$ investigou as habilidades de leitura e de escrita de palavras reais e de não-palavras isoladas entre crianças da $1^{\mathrm{a}}$ à $4^{\mathrm{a}}$ série de uma escola particular. Em relação à leitura de palavras reais, os resultados mostraram que, tanto em termos de precisão quanto de tempo de processamento, houve um efeito da frequência e da escolarização na leitura das crianças, tendo ocorrido também uma interação entre estes fatores. Tais resultados indicam, respectivamente, que houve uma influência lexical na leitura, que houve um ganho em proficiência e rapidez na leitura com a escolarização e que a frequência das palavras afetou de forma diferente a leitura das crianças das séries iniciais e das finais. Em relação ao efeito de regularidade, este se restringiu às séries iniciais e ao tempo de processamento das palavras pouco frequentes, o que foi interpretado como uma influência da regularidade somente entre esses estímulos. O comprimento das palavras também só afetou o tempo de reação, e em maior magnitude entre as crianças das séries iniciais ${ }^{3}$. Como conclusão do estudo, a autora aponta a 
identificação da existência dois processos distintos que se desenvolviam de maneira paralela, um lexical e outro fonológico, trazendo, assim, evidências de que o modelo de dupla-rota poderia ser estendido para a leitura do português brasileiro.

Os achados anteriores, no que tange ao efeito de frequência na precisão, foram replicados por um estudo conduzido por Guimarães (2004). A autora, utilizando a mesma lista de Pinheiro (1995), estudou um grupo de crianças com dificuldades de aprendizagem cursando a $3^{\mathrm{a}}$ e $4^{\mathrm{a}}$ séries do Ensino Fundamental, tendo como controle dois grupos de crianças com desenvolvimento normal da leitura, um deles pareado em termos da idade cronológica e outro composto de crianças mais jovens pareadas quanto à idade de leitura. Os resultados mostraram que houve um efeito geral de frequência e que este efeito foi maior na leitura do grupo de leitores mais jovens e do grupo que apresentava dificuldades de aprendizagem. Este achado, que está de acordo com a literatura, aponta para o surgimento de representações lexicais mais fortes nas fases mais adiantadas da aprendizagem da leitura entre crianças normais. Não foram reportadas informações sobre o efeito de regularidade.

Em relação ao estudo de Pinheiro (1995, 2008), duas considerações devem ser feitas. Primeiro, tanto para a avaliação da leitura, quanto da escrita, a classificação de regularidade das palavras considerou a relação fonemagrafema, ou seja, a direção da escrita. Para Pinheiro e Rothe-Neves (2001), tal fato pode ter induzido à ausência do efeito de regularidade nos erros, pois, conforme lembram os autores, muitas palavras irregulares para a escrita são regulares para a leitura (como cidade), ou seja, podem não gerar erros de pronúncia mesmo se lidas pela rota fonológica. Outro ponto que merece destaque é a metodologia utilizada no estudo, no qual foram considerados estatisticamente válidos apenas os resultados que se mostraram significativos tanto na análise de itens quanto na análise de sujeitos. Tal medida pode ter levado a divergências de resultados com outros trabalhos. Por exemplo, A. G. S. Capovilla et al. (1998), utilizando a mesma lista de palavras de Pinheiro (1995, 2008), com a diferença de apenas três itens, ao contrário desta autora, encontraram o efeito de regularidade nos erros cometidos na leitura. No entanto, como ressaltam Pinheiro e Rothe-Neves (2001), os referidos autores utilizaram apenas análises de sujeitos para reportar o efeito, o que contraria as recomendações de Clark (1973) - e de outros autores, como Raaijmakers (2003) e Raaijmakers,

escocesas equiparadas em termos de idade e desempenho na leitura. A autora demonstrou que a leitura competente de falantes de ambas as línguas foi semelhante no que se refere ao tempo de processamento e precisão para palavras reais e não-palavras e diferenciou-se principalmente em termos do efeito de regularidade (ausente na leitura competente em português) e de comprimento para palavras reais (maior em português do que em inglês).
Schrijnemakers e Gremmen (1999, 2006). Segundo Clark (1973), para que os resultados sejam generalizáveis tanto para a população de sujeitos a qual a amostra representa, quanto para a população de itens do qual o conjunto de itens experimentais foi derivado, é necessário que se encontre resultados significativos em ambos os tipos de análise, de sujeito e de itens ${ }^{4}$. Isso implica que os resultados de A. G. S. Capovilla et al. (1998) só podem ser generalizados com segurança para a população de sujeitos, não se estendendo para o corpus de palavras que dispomos na língua. Com exceção dos estudos de C. Justi e Justi (2009) e de Pinheiro, todos os resultados a serem relatados nesta revisão foram obtidos apenas com a análise de sujeitos, sendo, portanto as suas generalizações de alcance limitado ${ }^{5}$.

Em um estudo com crianças portuguesas, Sucena e Castro (2005) avaliaram a leitura de palavras reais e de não-palavras feita por crianças cursando da $1^{\mathrm{a}}$ à $4^{\mathrm{a}}$ série $(N=99)$ com o objetivo de investigar o efeito da consistência ortográfica na precisão e no tempo de processamento na leitura e determinar as estratégias preferenciais (lexicais ou fonológicas) utilizadas pelos participantes. As palavras do estudo foram divididas em quatro condições ortográficas, podendo ser simples (relação letra-som unívoca), complexas (formadas por grafemas compostos por mais de uma letra), regidas por regras contextuais e irregulares. Entre as não-palavras, somente não havia a condição irregular. Entre outras conclusões, o estudo mostrou a presença dos efeitos de regularidade (palavras regulares versus irregulares), de complexidade ortográfica (grafemas simples versus compostos) e de lexicalidade (palavras versus não-palavras) na leitura, tanto em termos de tempo de reação quanto de precisão. Além disso, o efeito de extensão apareceu a partir da $2^{\mathrm{a}}$ série no tempo de processamento de palavras e de não-palavras, tendo sido maior entre estas últimas. Os autores concluíram que as crianças utilizavam paralelamente tanto as estratégias lexicais (efeito lexical) quanto e fonológicas (efeitos de regularidade, extensão e complexidade) na leitura, o que aponta para um léxico ortográfico em desenvolvimento mesmo entre as crianças da $4^{a}$ série. Os resultados desse estudo estão em consonância com os resultados obtidos com crianças brasileiras (Pinheiro, 1995, 2008; Salles \& Parente, 2002).

Em um estudo de validação e normatização de uma bateria de avaliação da compreensão da leitura, Viana et 
al. (2003) investigaram o desempenho na leitura de 100 crianças portuguesas de $2^{\mathrm{a}}$ e $4^{\mathrm{a}}$ série $(50$ crianças por série). A tarefa de leitura em voz alta era composta por palavras que variavam quanto à frequência de ocorrência (alta e baixa), quanto ao comprimento (palavras grandes e pequenas) e quanto à lexicalidade (palavras reais e nãopalavras), mas não foi controlado o nível de regularidade dos itens. Além disso, o controle da frequência dos itens não foi feita com base em uma contagem específica para o vocabulário infantil. Como resultado, as autoras não encontraram o efeito de escolaridade na precisão na leitura de palavras reais e de não-palavras. Este resultado vai de encontro ao reportado por Sucena e Castro (2005), que demonstraram que somente na $4^{\mathrm{a}}$ série as crianças atingem níveis mais elevados de correção na leitura de palavras irregulares e de não-palavras com estrutura silábica mais complexa. $\mathrm{O}$ controle inadequado das variáveis psicolinguísticas no estudo de Viana et al. (2003) pode ter sido responsável pela divergência de resultados.

Com o objetivo avaliar as rotas preferenciais (lexical ou fonológica) utilizadas por crianças de $2^{\mathrm{a}}$ e $3^{\mathrm{a}}$ série de escolas particulares, Salles e Parente (2002) investigaram a emergência dos efeitos de frequência, regularidade, extensão e lexicalidade na leitura de palavras isoladas utilizando uma classificação que considera a regularidade para a leitura das palavras. Os resultados mostraram que a maioria das crianças da $3^{\text {a }}$ série utilizava ambas as rotas com proficiência, enquanto que as crianças da $2^{\text {a }}$ série eram preferencialmente leitoras fonológicas. Este resultado é condizente com a ideia de que, com o desenvolvimento, a leitura fonológica evolui para uma leitura lexical, mas que um bom leitor é proficiente no uso de ambas as rotas (Share, 1995). Em relação aos efeitos na leitura, o maior efeito encontrado foi o de regularidade, seguido do de extensão (na leitura de não-palavras) e de frequência, enquanto que o efeito de lexicalidade foi quase inexistente. Resultados semelhantes foram obtidos por Salles e Parente (2007), que estudaram uma amostra de crianças cursando a $2^{\mathrm{a}}$ série, desta vez oriunda de escolas públicas. Neste caso, foram relatados os efeitos de regularidade, frequência e extensão (na leitura de nãopalavras), mas novamente o efeito lexical não foi encontrado.

A presença do efeito de regularidade no trabalho de Salles e Parente $(2002,2007)$ reforça a ideia de que este efeito pode ser obtido considerando-se a regularidade grafema-fonema das palavras. No entanto, os resultados destes estudos devem ser considerados com cautela, uma vez que nestes trabalhos somente foram relatados os tamanhos dos efeitos em termos de diferenças de médias, não tendo sido conduzidas análises estatísticas para se verificar se tais diferenças foram significativas. Mesmo assim, pode-se afirmar que o tamanho das diferenças de médias encontrado aponta para tal direção.

Um fato que chama a atenção nos trabalhos supracitados é a ausência do efeito lexical, constantemente relatado tanto na língua inglesa (p. ex., Kinoshita, Lupker, \&
Rastle, 2004; McCann \& Besner, 1987; Rastle \& Coltheart, 1999) quanto no português (A. G. S. Capovilla et al., 1998; Godoy, 2005; Pinheiro, 1995, 2008; Sucena \& Castro, 2005). Duas breves considerações serão feitas para tentar explicar esses resultados, ambas de caráter metodológico. A primeira consideração refere-se à metodologia de apresentação dos estímulos, enquanto que a segunda diz respeito à metodologia de comparação das variáveis (no caso, palavras reais de diferentes níveis de frequência e não-palavras).

Em relação à apresentação dos estímulos, nos trabalhos Salles e Parente $(2002,2007)$, as palavras reais e as não-palavras foram apresentadas conjuntamente em uma mesma lista, ao invés de em listas separadas: uma contendo palavras reais de diferentes níveis de frequência, e outra contendo não-palavras. Este artifício pode ter induzido à ausência do efeito de lexicalidade. A explicação para este resultado está no efeito das estratégias utilizadas na leitura (Coltheart, 1978; Coltheart et al., 2001; Kinoshita et al., 2004). Assim, Coltheart (1978) demonstrou que a estratégia - lexical ou fonológica - escolhida pelo leitor pode ser afetada pelo tipo de estímulo - palavra real ou não-palavra - que está sendo lido. Nesse estudo, os participantes tinham que ler uma lista de nãopalavras pronunciáveis, com exceção do último item, que era uma palavra real altamente irregular (wolf). Vários dos participantes regularizaram esta palavra para rimar com a palavra golf, sendo que muitos deles sequer perceberam que havia uma palavra real na lista. Coltheart (1978) reporta esse achado como a influência do efeito da estratégia que é utilizada na leitura. Ele interpretou o resultado como sendo o efeito do "desligamento" da rota lexical pelos participantes, que utilizaram de forma prioritária a rota fonológica por esta gerar sempre a resposta correta na leitura das não-palavras. Em outros termos, a previsibilidade de que o próximo estímulo seria também uma não-palavra forçou a leitura dos estímulos da lista por meio da rota fonológica.

Desta forma, fato semelhante pode ter acontecido entre os participantes das pesquisas de Salles e Parente $(2002,2007)$. Como as palavras reais e as não-palavras foram reportadas em uma mesma lista, os participantes podem ter sido induzidos a utilizar a mesma estratégia para ler ambos os tipos de estímulos, a saber, a estratégia fonológica, dado que esta poderia gerar pronúncias plausíveis para a maioria dos estímulos. Nos trabalhos de Salles e Parente (2007), além da ausência do efeito de lexicalidade, o efeito das estratégias ficou evidente com o melhor desempenho das crianças na leitura de não-palavras em relação às palavras irregulares. Esse fato ilustra muito bem como a metodologia utilizada pelo pesquisador pode interferir na produção de efeitos que são utilizados para testar o modelo de processamento cognitivo da leitura e, consequentemente, na interpretação dos resultados.

Do ponto de vista do método de comparação das variáveis, o efeito lexical nesses trabalhos pode ter sido 
anulado em função de as não-palavras terem sido contrastadas com as palavras reais de ambas as frequências juntas, e não separadamente. Os estudos em português apontam para o fato de que a frequência de ocorrência da palavra interfere na produção desse efeito, pelo menos em uma lista com a classificação de regularidade do ponto de vista da leitura ${ }^{6}$. Por exemplo, Godoy (2005) demonstrou uma superioridade lexical na precisão e na velocidade de processamento da leitura de palavras de alta frequência em relação às não-palavras, mas o mesmo efeito não apareceu entre as palavras de baixa frequência (de fato, houve um efeito marginalmente significativo na precisão a favor das não-palavras, $\operatorname{com} p=0,65$. Esse resultado é esperado considerando a fase de desenvolvimento da leitura das crianças testadas (alunos da pré-escola e da $1^{\text {a }}$ série) e o tipo de classificação de regularidade de palavras utilizadas (palavras regulares e irregulares para leitura)). Desta forma, é esperado que o contraste entre as não-palavras e as palavras de alta frequência produza efeitos maiores do que o contraste feito com as palavras de baixa frequência. No último caso, a permanência de um efeito lexical reduzido (ou mesmo da ausência deste efeito) nas séries mais avançadas pode ser um indício de que as representações lexicais do leitor não estão sendo formadas de maneira adequada.

Outro estudo que merece consideração do ponto de vista metodológico é o trabalho recente desenvolvido por C. Justi e Justi (2009). Os autores avaliaram os efeitos de regularidade, lexicalidade e frequência nos erros e no tempo de resposta na leitura em voz alta de crianças de $3^{a} \mathrm{e}$ $4^{a}$ série do Ensino Fundamental. O tempo de resposta utilizado consistiu a lacuna de tempo entre a apresentação do estímulo à criança e o término da resposta (englobando, segundo os autores, as medidas de reação locucional e duração locucional reportadas por F. C. Capovilla, Macedo, Duduchi, \& Sória, 1997). Como resultado, encontrou-se os efeitos de lexicalidade e de frequência, tanto no tempo de resposta quanto nos erros produzidos, mas não o de regularidade.

A questão metodológica a ser apontada no trabalho de C. Justi e Justi (2009) refere-se ao fato de os autores terem usado como variável independente o Tempo de Duração da Resposta (TDR) e não a medida clássica de Tempo de Reação (TR), o que limita as comparações do referido estudo com os demais em português que usam o TR, embora concordemos que o trabalho de F. C. Capovilla et al. (1997) tenha demonstrado que as medidas locucionais são úteis para a avaliação da leitura. No entanto, a reserva que se faz aqui se deve a outra razão além da dificuldade de comparação do trabalho de C. Justi e Justi (2009)

${ }^{6}$ Pinheiro (2008) encontrou o efeito geral de lexicalidade no tempo de reação e na precisão no contraste entre palavras de alta e de baixa frequência com não-palavras, mas conforme visto anteriormente este estudo utilizou uma classificação de palavra diferente dos estudos que estamos tratando. com outros produzidos. Acreditamos que o TR e o TDR sejam indicadores de diferentes processos de acordo com modelo de dupla-rota, principalmente no português que é uma língua mais regular e mais previsível que o inglês, por exemplo. Consideremos a seguinte situação: a criança A, uma leitora competente, lê uma palavra irregular com baixo TR e com baixa duração locucional, produzindo um baixo TDR. As crianças B e C, duas leitoras não-competentes, lêem a mesma palavra de formas diferentes. A criança B silaba implicitamente a palavra e dá sua resposta, suponhamos que correta ${ }^{7}$ (produzindo alto TR e baixo TDR). A criança C, por sua vez, silaba explicitamente e, portanto, lê a palavra incorretamente. Assim, se considerássemos o TR, o efeito de regularidade estaria ausente para a criança A (por ter baixo TR) e presente no tempo de processamento na criança $\mathrm{B}$ (alto TR, baixo TDR). $\mathrm{Na}$ criança $\mathrm{C}$ o efeito se manifesta apenas nos erros, já que os tempos de resposta dos itens errados não são considerados nas análises estatísticas.

No entanto, considerando-se o TDR, o efeito de regularidade estaria ausente na leitura das crianças A e B, mas por razões diferentes. Enquanto que na criança A a ausência do referido efeito parece genuína (por o baixo TDR estar associado a um baixo TR) na criança $B$, a ausência desse efeito parece mais o resultado de um artefato metodológico. Dito em outras palavras, o uso do TDR, sem considerar a sua relação com o TR, pode forçar a ausência do efeito de regularidade entre os leitores pouco competentes e que tiram vantagens da silabação implícita, gerando um efeito cumulativo que anularia as diferenças entre as palavras regulares e irregulares. Esperamos com esse exercício ter demonstrado que o controle dos tempos de duração das respostas é relevante, todavia, os resultados dessa medida devem sempre interpretados em conjunção com os resultados dos tempos de reação.

O que dissemos acima constitui uma tentativa de explicação para a ausência do efeito de regularidade apenas no TDR no trabalho de C. Justi e Justi (2009), não se estendendo esta explicação aos erros produzidos pela amostra. Conforme dissemos, os autores também não encontraram o efeito de regularidade na precisão. No entanto, cabe ressaltar que, conforme lembram próprios autores, o estudo foi realizado com crianças das séries finais do Ensino Fundamental sendo que vários trabalhos têm relatado que nestas séries o efeito de regularidade tende a ser reduzido ou mesmo a desaparecer (p. ex.: Pinheiro, 1995; Pinheiro, Lúcio, \& Silva, 2008; Sucena \& Castro, 2005).

Em um estudo longitudinal, Godoy (2005) avaliou a influência do método de ensino (fônico versus global) no desenvolvimento de estratégias de processamento na lei-
${ }^{7}$ O que tem alta probabilidade de ocorrer, dado o caráter
quase previsível das palavras no português, mesmo no
caso das irregulares. Para uma discussão, ver C. Justi e
Justi (2009) e Lúcio (2008). 
tura e na escrita de crianças cursando da pré-escola à $1^{\mathrm{a}}$ série em escolas particulares de Florianópolis. Na Fase 3 do estudo, em que as crianças cursavam a $1^{\mathrm{a}}$ série, e utilizando uma lista experimental de palavras contrastadas quanto à frequência, regularidade para a leitura e extensão (Pinheiro, 2003), a autora descobriu, além do efeito lexical supracitado, a presença de um efeito de extensão e de frequência na precisão e na velocidade de leitura de ambos os grupos de crianças. $\mathrm{O}$ efeito de regularidade se restringiu à precisão na leitura, mas pôde ser obtido para o tempo de reação quando valores extremos (outliers) foram retirados da análise. Tais resultados confirmam o uso do processo fonológico por ambos os grupos de crianças, mas também apontam para a formação paralela de um processo lexical. Apesar de nesta fase do estudo as crianças expostas ao método fônico terem apresentado um melhor resultado do que as expostas pelo método global, não foi encontrado um efeito geral do método de alfabetização na leitura. Este resultado sugere que, independentemente do método de ensino, o processo de decodificação pode ser alcançado com eficiência em uma ortografia transparente, como é o caso do português. No entanto, não se descarta a hipótese de que o contraste entre os métodos de alfabetização feitos pelo estudo não tenha sido suficientemente forte para demonstrar o efeito do método na leitura (Godoy, 2005; Godoy, Defior, \& Pinheiro, 2007).

Os resultados de Godoy para crianças brasileiras de $1^{a}$ série estão de acordo com os encontrados por Cary e Vale (1997) com crianças portuguesas da mesma faixa etária (ver também Vale \& Bertelli, 2001). O estudo tinha como objetivo investigar se as habilidades de detectar unidades sub-silábicas no jardim de infância tem um valor preditivo para o desempenho posterior na leitura. Vinte e cinco crianças leram em voz alta 24 palavras divididas em termos de frequência (alta e baixa), regularidade (regular e irregular) e tamanho (grandes e compridas). Os autores relataram um forte efeito de regularidade na precisão na leitura das crianças, indicando uso de estratégia de mediação fonológica na leitura, o que é esperado de acordo com a idade dos participantes. Todavia, os autores não informaram a direção da classificação de regularidade adotada no trabalho.

Em um estudo recente, Stivanin e Scheuer (2008) estabeleceram uma comparação entre o tempo de latência na leitura e na nomeação de objetos feita por estudantes de $2^{\mathrm{a}}$ à $4^{\mathrm{a}}$ série da cidade de São Paulo. Em relação à tarefa de leitura em voz alta, os autores dividiram os estímulos em duas categorias de regularidade (regular versus irregular) e em diferentes tamanhos (4 a 6 grafemas) e estruturas silábicas (consoante-vogal (CV) ou outras estruturas), criando conjuntos de estímulos que diferiam em termos de complexidade, variando de uma estrutura mais simples (4 grafemas, estrutura CV e regulares) a uma mais complexa (6 grafemas, outras estruturas silábicas, irregulares). Os resultados apontaram para a presença de um efeito de frequência ${ }^{8}$, regularidade e de extensão. No entanto, no que se refere aos efeitos de frequência e de regularidade, podemos registrar peculiaridades importantes. Primeiramente, tomando o efeito de frequência, este ocorreu de forma atípica na leitura das crianças de $2^{\mathrm{a}}$ série, que apresentaram maior tempo de latência para as palavras alta frequência do que para as de baixa frequência (ver também Stivanin \& Scheuer, 2007).

Em relação ao efeito de regularidade, nas palavras de baixa frequência, foi restrito à leitura das crianças mais jovens ( $2^{a}$ série), o que está em consonância com a conclusão de que o efeito de regularidade reduz sua força à medida que a escolaridade avança. No entanto, este resultado é contraditório com o fato de que entre as palavras de alta frequência, em que não se espera encontrar nenhum efeito de regularidade, mesmo nos leitores iniciantes, ele tenha ocorrido em todas as séries nas palavras com maiores níveis de complexidade. Uma tentativa de explicação seria a de que este efeito foi restrito às comparações feitas em termos de nível de complexidade, sendo possível a ausência do efeito considerando-se as latências nos estímulos de uma maneira geral. Entretanto, as autoras não reportaram resultados gerais dos efeitos em suas análises estatísticas, assim como não apresentam a lista de palavras testada. Mediante a última omissão, só nos resta contemplar a possibilidade de que um pareamento inadequado entre as palavras complexas de alta e de baixa frequência de ocorrência tenha sido feito, principalmente conhecendo-se quão limitado é o universo de palavras de alta frequência.

Com o objetivo de avaliar o impacto da relação grafema-fonema e fonema-grafema na emergência do efeito de regularidade na leitura, Pinheiro, Lúcio e Silva (2008) conduziram um estudo no qual o nível de regularidade das palavras foi controlado tanto do ponto de vista da leitura quanto da escrita. Assim, um grupo de crianças da $1^{\mathrm{a}}$ à $3^{\mathrm{a}}$ série de uma escola particular leu um corpo de 323 palavras de baixa frequência de ocorrência (Pinheiro, 1996, 2004). Conforme esperado a partir das recomendações de Parente, Hosogi e Lecours (1997) e de Pinheiro e Rothe-Neves (2001), controlando-se o nível de regularidade das palavras para a leitura, Pinheiro, Lúcio e Silva (2008) encontraram um efeito de regularidade na precisão. No entanto, e ao contrário dos achados anteriores de Pinheiro (1995, 2008), em que as palavras da lista testada foram classificadas na direção fonema-grafema, o efeito não se estendeu ao tempo de reação. O efeito no

\footnotetext{
${ }^{8}$ Com exceção dos contrastes feitos entre as palavras mais simples de alta frequência versus palavras simples de baixa frequência e também nos contrastes entre as palavras mais complexas nos mesmos níveis de frequência. Isto pode ter ocorrido porque as palavras simples, independentemente do seu nível de frequência, foram lidas pelas crianças de maneira rápida, enquanto que o contrário ocorreu com as palavras mais complexas, que levaram mais tempo para serem lidas.
} 
tempo de processamento só foi observado quando se considerou a classificação de regularidade das palavras para a escrita, o que está em consonância com os resultados de Pinheiro (1995, 2008), mas diferentemente destes, o efeito também se estendeu aos erros produzidos. Como explicação, supôs-se que a classificação de palavras utilizada no estudo de Pinheiro, Lúcio e Silva (2008) (que considerou apenas dois níveis de regularidade, tanto para a leitura quanto para a escrita) possa ter interferido nos resultados. Outra possibilidade seria a de que o efeito de regularidade na precisão tenha ocorrido em função da grande diferença em termos de número de palavras utilizadas nos dois estudos. Enquanto a lista de palavras da pesquisa original era composta de 96 palavras, a da pesquisa sob consideração era formada de 323 palavras, o que pode ter induzido as crianças a errarem mais como resultado de fadiga (possibilidade não testada).

Focando nossa atenção para o efeito de regularidade, os resultados encontrados em todos os estudos citados acima são resumidos na Tabela $1^{9}$. Pela análise desta tabela, podemos observar que é plausível a hipótese levantada por Pinheiro, Lúcio e Silva (2008) de que a emergência do efeito de regularidade na leitura possa variar de acordo com a classificação de regularidade das palavras (direção da leitura ou da escrita). Assim, podemos ver que, independentemente do número de categorias de regularidade utilizadas no estudo, quando se considera a regularidade para a leitura das palavras, o efeito de regularidade está sempre presente na precisão ${ }^{10}$.
De maneira semelhante, e novamente independente da quantidade de categorias, utilizando-se a classificação de regularidade para a escrita das palavras, o efeito está sempre presente no tempo de processamento na leitura. Isso conduz à hipótese de que o efeito de regularidade na leitura aparecerá na precisão, sempre que o nível de regularidade das palavras for classificado quanto à direção da leitura, enquanto que, para o tempo de processamento, esse efeito sempre aparecerá quando a classificação para a escrita é considerada.

No entanto, um fato complicador é que o surgimento do efeito para a precisão e para o TR parece variar em relação ao número de categorias utilizadas no estudo quando se considera, respectivamente, a classificação das palavras para a escrita ou para a leitura. Desta forma, nos estudos de Godoy (2005) e de Pinheiro, Lúcio e Silva (2008), em que se consideraram apenas duas categorias, o efeito não apareceu no tempo de reação controlando-se o nível de regularidade das palavras para a leitura ${ }^{11}$, mas o contrário ocorreu no trabalho de Sucena e Castro (2005), que utilizaram quatro categorias diferentes. Em relação à precisão, Pinheiro $(1995,2008)$, utilizando a classificação para a escrita e três categorias de regularidade, encontrou o efeito de regularidade apenas para o grupo de sujeitos, mas não para os itens (os resultados foram replicados por A. G. S. Capovilla et al. (1998) também na análise de sujeitos). No entanto, Pinheiro, Lúcio e Silva (2008) encontraram o efeito tanto para os itens quanto para os sujeitos, utilizando apenas duas categorias de regularidade.

Tabela 1

Resultados Para o Efeito de Regularidade na Leitura em Diversos Estudos com a Língua Portuguesa, em Função da Direção da Classificação de Regularidade das Palavras, e Estatísticas por eles Utilizadas

\begin{tabular}{|c|c|c|c|c|c|}
\hline Estudo & $\begin{array}{c}\text { Direção da } \\
\text { Classificação } \\
\text { de regularidade }\end{array}$ & $\begin{array}{l}\text { Número de } \\
\text { categorias } \\
\text { (precisão) }\end{array}$ & $\begin{array}{l}\text { Efeito de } \\
\text { regularidade } \\
\text { (precisão) }\end{array}$ & $\begin{array}{l}\text { Efeito de } \\
\text { regularidade } \\
\text { (TR) }\end{array}$ & $\begin{array}{c}\text { Estatística } \\
\text { utilizada }\end{array}$ \\
\hline A. G. S. Capovilla et al. (1998) & Escrita & Três & Presente & Presente & $\operatorname{ANOVA}(F 1)$ \\
\hline Godoy $(2005)$ & Leitura & Duas & Presente & Ausente & $\operatorname{ANOVA}(F 1)$ \\
\hline C. Justi e Justi (2009) & Leitura & Duas & Ausente $^{1}$ & Ausente $^{1,2}$ & $\min F^{\prime}$ \\
\hline \multirow[t]{2}{*}{ Lúcio et al. (2010) } & Leitura & Quatro & Presente & Ausente & GEE \\
\hline & Escrita & Quatro & Presente & Presente & GEE \\
\hline Pinheiro $(1995,2008)$ & Escrita & Três & Ausente & Presente & $\operatorname{ANOVA}(F 1$ e $F 2)$ \\
\hline \multirow[t]{2}{*}{ Pinheiro, Lúcio e Silva (2008) } & Leitura & Duas & Presente & Ausente & $\min F^{\prime}$ \\
\hline & Escrita & Duas & Presente & Presente & $\min F^{\prime}$ \\
\hline Salles (2005) & Leitura & Duas & Presente & - & - \\
\hline Salles e Parente $(2002,2007)$ & Leitura & Duas & Presente & & \\
\hline Stivanin e Scheuer, 2008 & Leitura & Duas & - & Presente $^{3}$ & ANOVA \\
\hline Sucena e Castro (2005) & Leitura & Quatro & Presente & Presente & $\operatorname{ANOVA}(F 1)$ \\
\hline
\end{tabular}

Nota TR $=$ Tempo de reação. ${ }^{1} \mathrm{O}$ estudo considerou a leitura crianças de $3^{\mathrm{a}} \mathrm{e} 4^{\mathrm{a}}$ séries. ${ }^{2} \mathrm{O}$ estudo considerou o tempo de duração da resposta e não o tempo de reação. ${ }^{3}$ Efeito presente apenas na leitura de crianças de $2^{\mathrm{a}}$ série e em palavras complexas de alta frequência.

${ }^{9}$ Somente os estudos que reportam dados sobre o efeito
de regularidade.
${ }^{10}$ Ver considerações sobre o estudo de C. Justi e Justi
(2009) anteriormente no texto, que avaliaram crianças das séries finais ( $3^{\mathrm{a}}$ e $4^{\mathrm{a}}$ séries) do Ensino Fundamental e não encontraram o efeito de regularidade.

${ }^{11}$ Ver as considerações feitas sobre o estudo de Stivanin e Scheuer (2008) anteriormente no texto. 
A hipótese de que o número de categorias de regularidade poderia interferir na emergência do efeito de regularidade foi testada por Lúcio et al. (2010). As autoras, utilizando um modelo de regressão hierárquica (método de Estimação de Equação Generalizada), dividiram as palavras do estudo (as mesmas de Pinheiro, Lúcio, \& Silva, 2008) em duas ou quatro categorias de regularidade grafofonêmicas e fonografêmicas ${ }^{12}$. Como resultado, as autoras descobriram que o uso de quatro categorias de regularidade foi mais eficaz para explicar o desempenho das crianças do que o uso de apenas duas categorias. Nesse estudo, o efeito de regularidade foi encontrado no tempo de reação considerando-se apenas a relação fonografêmica das palavras, de maneira semelhante ao que foi encontrado por Pinheiro (1995, 2008); mas diferentemente desta, e de maneira semelhante a Pinheiro, Lúcio e Silva (2008), o efeito foi encontrado na precisão considerando-se ambas as direções de classificação. As diferenças de desempenho foram maiores entre as palavras que apresentavam regras contextuais concomitantes a uma irregularidade (palavras irregulares-regras (Pinheiro, 2007a)) em relação às demais. Estas parecem ser as palavras mais difíceis para as crianças cursando as séries iniciais do Ensino Fundamental. Dito isto, o uso de listas de palavras com classificação de regularidade que permitem a averiguação do efeito de regularidade tanto do ponto de vista da leitura quanto da escrita (nos moldes introduzidos por Pinheiro, 2007a, 2007b e testados por Lúcio et al., 2010 e Pinheiro, Lúcio, \& Silva, 2008) parece ser uma solução para a estimação do efeito de regularidade em nosso idioma.

\section{Discussão}

Tendo em vista o que foi exposto a respeito do estudo de reconhecimento de palavras em português, particularmente no que diz respeito à tarefa de leitura em voz alta de palavras reais isoladas, podemos concluir três pontos importantes: (a) os diversos estudos em língua portuguesa avaliados confirmam, de uma maneira geral, os postulados do modelo de dupla-rota, segundo o qual a leitura pode ocorrer por meio de dois processos distintos e simultâneos, um lexical e outro fonológico; (b) as crianças brasileiras parecem utilizar preferencialmente a estratégia fonológica no início da aprendizagem da leitura, a qual vai sendo gradualmente substituída pelo uso prioritário estratégia lexical, que é mais eficaz do ponto de vista do desenvolvimento. $\mathrm{O}$ apego à estratégia fonológica nesse período parece ser devido ao caráter quase regular da língua portuguesa, que apresenta apenas dois casos de irregularidade para a leitura (Parente et al., 1997). No entanto, a influência do método de alfabetização utilizado nas escolas, que geralmente é calcado no ensino formal das relações letra-som aos leitores iniciantes, ainda não pode ser descartada; (c) questões metodológicas interferem nos resultados encontrados pelos diferentes pesquisadores, e essas questões esbarram em quatro pontos principais: a maioria dos pesquisadores que trabalham com o reconhecimento de palavras no Brasil tem desconsiderado que, em estudos que utilizam materiais linguísticos, é importante examinar a generalização dos resultados para o corpo de itens, além dos grupos de sujeitos, o que é obtido respectivamente pela presença de resultados significativos na análise de itens e na análise de sujeitos (ver nota.$^{\circ}{ }^{4}$ ); a forma de apresentação dos estímulos da tarefa pode inibir a manifestação do efeito de regularidade, devido ao efeito das estratégias (Coltheart et al., 2001); o número de categorias utilizadas para a classificação das palavras parece ser importante para a emergência do efeito de regularidade na leitura, apesar de essa influência ainda não estar claramente compreendida; parece haver uma diferença na influência do nível de regularidade para a leitura e para a escrita das palavras para a emergência do efeito de regularidade na precisão e no tempo de reação na leitura. Há consistentes indícios de que, tanto para o tempo de reação quanto para a precisão, a classificação fonografêmica possa ser utilizada, apesar de que, no caso da precisão, o efeito de regularidade mostra mais força quando a classificação grafofonêmica é considerada (Lúcio et al., 2010). Se o controle do nível de regularidade para a escrita mostrar-se de fato eficiente para a obtenção do efeito de regularidade na leitura (o que será confirmado por pesquisas posteriores), este será um achado importante para a pesquisa, por permitir a obtenção dos efeitos na precisão e no tempo de reação controlando-se apenas um nível de regularidade ${ }^{13}$. Estas considerações certamente trazem informações relevantes para as futuras pesquisas que busquem investigar o efeito de regularidade na língua portuguesa.

\section{Referências}

Capovilla, A. G. S., Capovilla, F. C., \& Macedo, E. C. (1998). Validação do software CronosFonos para a análise de tempo de reação, duração e frequência de segmentação locucionais na leitura em voz alta de itens isolados. Ciência Cognitiva: Teoria, Pesquisa e Aplicação, 2(3), 253-340.

Capovilla, A. G. S., \& Dias, N. M. (2007). Desenvolvimento de estratégias de leitura no ensino fundamental e correlação com nota escolar. Psicologia em Revista, 13(2), 363-382.

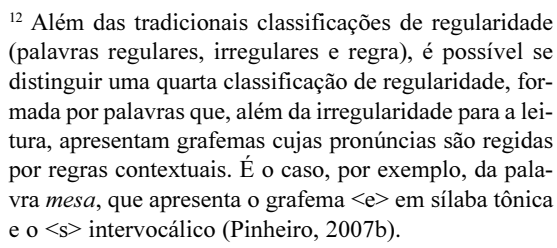

${ }^{12}$ Além das tradicionais classificações de regularidade (palavras regulares, irregulares e regra), é possível se distinguir uma quarta classificação de regularidade, formada por palavras que, além da irregularidade para a leitura, apresentam grafemas cujas pronúncias são regidas por regras contextuais. É o caso, por exemplo, da palavra $m e s a$, que apresenta o grafema $<\mathrm{e}>\mathrm{em}$ sílaba tônica e o $<$ s $>$ intervocálico (Pinheiro, 2007b).

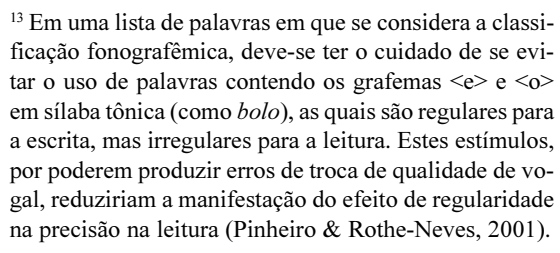

${ }^{13}$ Em uma lista de palavras em que se considera a classificação fonografêmica, deve-se ter o cuidado de se evitar o uso de palavras contendo os grafemas $<\mathrm{e}>\mathrm{e}<\mathrm{o}>$ em sílaba tônica (como bolo), as quais são regulares para a escrita, mas irregulares para a leitura. Estes estímulos, por poderem produzir erros de troca de qualidade de vogal, reduziriam a manifestação do efeito de regularidade na precisão na leitura (Pinheiro \& Rothe-Neves, 2001). 
Capovilla, F. C., \& Capovilla, A. G. S. (1996). Leitura, ditado e manipulação fonêmica em função de variáveis psicolinguísticas em escolares de terceira a quinta série com dificuldades de aprendizagem. Revista Brasileira de Educação Especial, 2(4), 53-71.

Capovilla, F. C., Macedo, E., Duduchi, M., \& Sória, R. (1997). Análise computadorizada de leitura em voz alta via rotas fonológica e lexical. Ciência Cognitiva: Teoria, Pesquisa e Aplicação, 1, 81-140.

Cary, L., \& Vale, A. P. (1997, September). Predicting portuguese first graders reading ability from sensitivity to subsyllabic units assessed in kindergarten. Paper presented at the 4th European Conference on Psychological Assessment, Lisbon, Portugal. Retrieved July 17, 2009, from http://home.utad.pt/ $\sim$ ple/?pagina $=$ content/divulgacao\#publicacoes

Clark, H. (1973). The language-as-fixed-effect fallacy: A critique of language statistics in psychological research. Journal of Verbal Learning and Verbal Behavior, 12, 335-359.

Coltheart, M. (1978). Lexical access in simple reading tasks. In G. Underwood (Ed.), Strategies of information processing (pp. 151-216). London: Academic Press.

Coltheart, M., Rastle, K., Perry, C., Langdon, R., \& Ziegler, J. (2001). DRC: A dual route cascaded model of visual word recognition and reading aloud. Psychological Review, 108(1), 204-256.

Godoy, D. M. A. (2005). Aprendizagem inicial da leitura e da escrita no português do Brasil: Influência da consciência fonológica e do método de alfabetização. Tese de Doutorado não-publicada, Universidade Federal de Santa Catarina, Florianópolis, SC.

Godoy, D., Defior, S., \& Pinheiro, A. M. V. (2007). Impacto do método de alfabetização sobre o desenvolvimento da consciência fonêmica, da leitura e da escrita no português do Brasil. Educação. Temas e Problemas, 4(2), 75-95.

Guimarães, S. R. K. (2004). O papel das pistas do contexto verbal no reconhecimento de palavras. Psicologia em Estudo (Maringá), 9(2), 279-289.

Justi, C., \& Justi, F. (2009). Os efeitos de lexicalidade, frequência e regularidade na leitura de crianças falantes do português brasileiro. Psicologia: Reflexão e Crítica, 22(2), 163-172.

Justi, F., \& Pinheiro, A. M. V. (2006). O efeito de vizinhança ortográfica no português do Brasil: Acesso lexical ou processamento estratégico? Revista Interamericana de Psicología, 40(3), 257-280.

Kinoshita, S., Lupker, S. J., \& Rastle, K. (2004). Modulation of regularity and lexicality effects in reading aloud. Memory \& Cognition, 32(8), 1255-1264.

Lúcio, P. S. (2008). Investigação psicométrica de uma tarefa de leitura em voz alta de palavras isoladas. Dissertação de Mestrado não-publicada, Universidade Federal de Minas Gerais, Belo Horizonte, MG.

Lúcio, P. S., Pinheiro, A. M. V., \& Nascimento, E. (2010). A influência de fatores sociais, individuais e linguísticos no desempenho de crianças na leitura em voz alta de palavras isoladas. Psicologia: Reflexão e Crítica, 23(3), 496-505.

Macedo, E. C., Lukasova, K., Yokomizo, J. E., Ariente, L. C., Koakutu, J., \& Schwartzman, J. S. (2007). Processos perceptuais e cognitivos na leitura de palavras: Propriedades dos movimentos oculares. Revista Semestral da Associação Brasileira de Psicologia Escolar e Educacional (ABRAPEE), 11(2), 275-283.
McCann, R., \& Besner, D. (1987). Reading pseudohomophones: Implications for models of pronunciation assembly and the locus of word-frequency effects in naming. Journal of Experimental Psychology: Human Perception and Performance, 13(1), 14-24.

Parente, M. A. M. P., Hosogi, M. L., \& Lecours, A. R. (1997). Conduta clínica. In A. R. Lecours \& M. A. M. Parente (Eds.), Dislexia: Implicações do sistema de escrita do português (pp. 86-105). Porto Alegre, RS: Artes Médicas.

Pinheiro, A. M. V. (1989). Reading and spelling in Brazilian Portuguese. Unpublished doctoral dissertation, University of Dundee, Escócia.

Pinheiro, A. M. V. (1995). Reading and spelling development in Brazilian Portuguese. Reading and Writing: An Interdisciplinary Journal, 7, 111-138.

Pinheiro, A. M. V. (1996). Contagem de frequência de ocorrência de palavras expostas a crianças de $1^{a}$ à $4^{a}$ série do Ensino Fundamental. São Paulo, SP: Associação Brasileira de Dislexia.

Pinheiro, A. M. V. (1999). Cognitive assessment of competent and impaired reading in Scottish and Brazilian children. Reading and Writing, 11, 175-211.

Pinheiro, A. M. V. (2003). Classificação de regularidade de palavras (CNPq COCHS/DPH No. 168/03). Belo Horizonte, MG: Universidade Federal de Minas Gerais, Departamento de Psicologia.

Pinheiro, A. M. V. (2004). Banco de palavras de baixa frequência de ocorrência, para crianças brasileiras da $1^{a}$ à $4^{a}$ série do Ensino Fundamental, classificadas em termos de estrutura silábica, número de letras e regularidade para leitura e para escrita (FAPEMIG DC/SOT No. 1202/2004). Belo Horizonte, MG: Universidade Federal de Minas Gerais, Departamento de Psicologia.

Pinheiro, A. M. V. (2007a). Avaliação de competências psicológicas da população infanto-juvenil de Belo Horizonte: Inteligência e habilidades de leitura e escrita (FAPEMIG DC/ SOT No. 1806/2007). Belo Horizonte, MG: Universidade Federal de Minas Gerais, Departamento de Psicologia.

Pinheiro, A. M. V. (2007b). Banco de palavras de baixa frequência de ocorrência, para crianças brasileiras da $1^{\mathrm{a}}$ à $4^{\mathrm{a}}$ série do Ensino Fundamental, classificadas em termos de estrutura silábica, número de letras e regularidade para leitura e para escrita. In I. Sim-Sim \& F. L. Vianna, Para avaliação do desempenho da leitura. Portugal, Lisboa: Ministério da Educação.

Pinheiro, A. M. V. (2008). Leitura e escrita: Uma abordagem cognitiva (2. ed.). São Paulo, SP: Livro Pleno.

Pinheiro, A. M. V., Costa, A. E., \& Justi, F. (2005). Reconhecimento de palavras reais e de não-palavras em crianças de $1^{\text {a }}$ a $4^{\mathrm{a}}$ série: Uma tarefa de decisão lexical. Revista de Estudos da Linguagem, 13(2), 145-170.

Pinheiro, A. M. V., Lúcio, P. S., \& Cunha, R. C. (2008). Tarefa de leitura de palavras em voz alta: Uma proposta de análise de erros. Revista Portuguesa de Educação, 21(2), 115-138.

Pinheiro, A. M. V., Lúcio, P. S., \& Silva, D. M. R. (2008). O efeito de regularidade grafema-fonema e fonema-grafema na leitura em voz alta de palavras isoladas. Psicologia: Teoria e Prática, 10(2), 16-20.

Pinheiro, A. M. V., \& Rothe-Neves. (2001). Avaliação cognitiva da leitura: As tarefas de leitura em voz alta e ditado. Psicologia: Reflexão e Crítica, 14(2), 399-408. 
Lúcio, P. S. \& Pinheiro, A. M. V. (2011). Vinte Anos de Estudo sobre o Reconhecimento de Palavras em Crianças Falantes do Português: Uma Revisão de Literatura.

Raaijmakers, J. G. W. (2003) A further look at the "Languageas-Fixed-Effect Fallacy". Canadian Journal of Experimental Psychology, 57(3), 141-151.

Raaijmakers, J. G., Schrijnemakers, J. M. C., \& Gremmen, F. (1999). How to deal with "The language-as-Fixed-Effect Fallacy": Common misconceptions and alternative solutions. Journal of Memory and Language, 41, 416-426

Raaijmakers, J. G., Schrijnemakers, J. M. C., \& Gremmen, F. (2006). Corrigendum "How to deal with 'The language-asFixed-Effect Fallacy': Common misconceptions and alternative solutions" [Journal of Memory and Language, 41 (1999), 416-426]. Journal of Memory and Language, 54, 634.

Rastle, K., \& Coltheart, M. (1999). Serial and strategic effects in reading aloud. Journal of Experimental Psychology: Human Perception and Performance, 25, 461-481.

Salles, J. F. (2001). O uso das rotas de leitura fonológica e lexical em escolares: Relações com compreensão, tempo de leitura e consciência fonológica. Dissertação de Mestrado não-publicada, Universidade Federal do Rio Grande do Sul, Porto Alegre, RS

Salles, J. F. (2005). Habilidades e dificuldades de leitura e escrita em crianças de $2^{a}$ série: Abordagem neuropsicológica cognitiva. Tese de Doutorado não-publicada, Universidade Federal do Rio Grande do Sul, Porto Alegre, RS.

Salles, J. F., \& Parente, M. A. M. P. (2002). Processos cognitivos na leitura de palavras em crianças: Relações com compreensão e tempo de leitura. Psicologia: Reflexão e Crítica, 15(2), 321-331.

Salles, J. F., \& Parente, M. A. M. P. (2006). Heterogeneidade nas estratégias de leitura/escrita em crianças com dificuldades de leitura e escrita. Psico, 37(1), 83-90.

Salles, J. F., \& Parente, M. A. M. (2007). Avaliação da leitura e escrita de palavras em crianças de $2^{a}$ série: Abordagem neuropsicológica cognitiva. Psicologia: Reflexão e Crítica, 20(2), 220-228.

Share, D. L. (1995). Phonological recoding and self-teaching: Sine qua non of reading acquisition. Cognition, 55(2), 151218.

Sucena, A., \& Castro, S. L. (2005). Estratégias fonológicas e ortográficas na aprendizagem da leitura do português europeu. Anales de La Revista de Psicologia General y Aplicada, 10(3). Retrieved January 25, 2009, from http://www.fedap.es/ IberPsicologia/iberpsi10/congreso_lisboa/sucena/sucena.htm

Stivanin, L., \& Scheuer, C. (2007). Tempo de latência para a leitura: Influência da frequência da palavra escrita e da escolarização. Revista da Sociedade Brasileira de Fonoaudiologia, 12(3), 206-213.

Stivanin, L., \& Scheuer, C. (2008). Comparação do tempo de latência entre nomeação e leitura em escolares. Psicologia em Estudo (Maringá), 13(1), 89-96.

Viana, F. L., Pereira, I. S. P., \& Teixeira, M. M. V. C. (2003). A PROCOMLEI: Uma prova de avaliação da compreensão leitora. Revista Galego-Portuguesa de Psicoloxía e Educación, 10(8), 1-12.

Vale, A. P., \& Bertelli, R. (2001, May). Brincar com rimas no jardim de infância é divertido, mas... Paper presented at the $1^{\circ}$ Encontro de Educadores de Infância, Açores, Portugal. Retrieved July 17, 2009, from http://home.utad.pt/ ple/ ?pagina $=$ content/divulgacao\#publicacoes 